\title{
A Semi-Theoretical Equation for the Chain Dimension in Dilute Solutions and Its Experimental Verification, and the Coil-Globule Transition
}

\author{
Tomo-o OYAMA, Kohzoh SHIOKAWA, and Koumei BABA* \\ Department of Applied Science, Faculty of Engineering, Kyushu University, \\ Hakozaki, Higashi-ku, Fukuoka 812, Japan
}

(Received September 11, 1984)

\begin{abstract}
A semi-theoretical equation for the chain dimension in dilute solutions based on the perturbational mean field theory,$$
\alpha^{5}-\alpha^{3}=\tilde{v}_{0 \mathrm{M}}(1-\tilde{\theta} / T) M^{1 / 2}+\tilde{v}_{3}\left(\alpha^{-3}-1\right)
$$

was proposed $(\alpha$, the expansion factor; $\tilde{\theta}$, the temperature at which the second virial coefficient of polymers $A_{2}=0 ; \tilde{v}_{0 \mathrm{M}}$ and $\tilde{v}_{3}$, phenomenological constant; $M$, the molecular weight; $T$, the absolute temperature). In derivation of the equation, the difference between $\theta$ (the temperature at which the second virial coefficient of segments $v_{2}=0$ ) and $\tilde{\theta}$ was taken into the consideration. Experimental verification was done by the polystyrene-cyclohexane, methylcyclohexane, and benzene solutions. The equation represented the experimental results well in the wide range of $(1-\tilde{\theta} / T) M^{1 / 2}$. The sharpness of the coil-globule transition was discussed. It was concluded that the coil-globule transition is not a phase transition type but a cross-over type at least in the polystyrene-cyclohexane solution.
\end{abstract}

KEY WORDS Chain Dimension / Theta Temperature / Excluded Volume Effect / Expansion Factor / Polystyrene / Coil-Globule Transition /

In the polymer chain statistics, the theta temperature plays an important role. This temperature is defined as the temperature $(\theta)$ at which the second virial coefficient of segments $\left(v_{2}\right)$ vanishes. On the other hand, the theta temperature is experimentally determined as the temperature $(\tilde{\theta})$ at which the second virial coefficient of polymer molecules $\left(A_{2}\right)$ vanishes. The recent theories ${ }^{1,2}$ have pointed out the difference between these two theta temperatures. In the analysis of the experimental results, this difference must be taken into consideration.

In this report, we propose a semi-theoretical equation for the chain dimension $\left(\left\langle S^{2}\right\rangle\right)$ in dilute solutions. This equation is based on the perturbational mean field theory (PMFT) and includes a consideration on the difference between the two theta temperatures. Experimental results of polystyrene solutions are analysed by using this equation. Further, the coil-globule transition is discussed on the basis of our theoretical and experimental results.

\section{THEORETICAL BACKGROUND}

Results $^{3-5}$ by PMFT are slightly different one another, but the most of them can be summarized in the next equation.

$$
\alpha^{5}-\alpha^{3}=v_{2} N^{1 / 2}+v_{3} / \alpha^{3} \quad(N \gg 1),
$$

* Present Address: Department of Materials Science and Engineering, Faculty of Engineering, Nagasaki University, Nagasaki 852, Japan. 
where $\alpha^{2}=\left\langle S^{2}\right\rangle /\left\langle S^{2}\right\rangle_{0}\left(\left\langle S^{2}\right\rangle_{0}:\left\langle S^{2}\right\rangle\right.$ for the Gaussian chain), $v_{2}$ and $v_{3}$ are the dimensionless quantities proportional to the second and the third virial coefficients of segments, respectively, and $N$ is the degree of polymerization. In the derivation of eq 1 , virial coefficients higher than $v_{3}$ were neglected, since their order are $N^{-1 / 2}$ or less in the range of $T \geqq \theta$. In eq 1 , it is assumed that

$$
v_{2}=v_{0}(1-\theta / T)
$$

and

$$
v_{3}=\text { constant }(>0),
$$

where $T$ is the absolute temperature, and $v_{0}(>0)$ is the value of $v_{2}$ at $T \rightarrow \infty$. $\theta$ is eq 2 is the temperature at which $v_{2}=0$. Equation 1 includes only two positive dimensionless parameters, $v_{0}$ and $v_{3}$.

Curves for $\alpha^{2}$ vs. $v_{2} N^{1 / 2}$ calculated by eq 1 are shown in Figure 1. The numerals in the figure are the values of $v_{3}$. Equation 1 gives the next limiting relations,

$$
\begin{array}{ll}
\left\langle S^{2}\right\rangle \sim v_{2}^{2 / 5} N^{6 / 5} & \left(v_{2} N^{1 / 2} \gg 0\right), \\
\left\langle S^{2}\right\rangle \sim\left(1+v_{3}\right) N & \left(v_{2} N^{1 / 2}=0\right),
\end{array}
$$

and

$$
\left\langle S^{2}\right\rangle \sim\left(-v_{3} / v_{2}\right)^{2 / 3} N^{2 / 3} \quad\left(v_{2} N^{1 / 2} \ll 0\right) .
$$

These relations coincide with the DaoudJannink's relations ${ }^{6}$ based on the scaling theory. However, it must be noticed that this coincidence is a result from a remarkable cancellation of two errors in MFT. ${ }^{1}$

As shown in the Figure 1, the value of $\alpha^{2}$ at $v_{2} N^{1 / 2}=0$ depends on the value of $v_{3}$. And as pointed out in the introductory section, $\theta$ can not be determined experimentally. Therefore, $\theta$ has not the character as a experimental standard temperature. The theta temperature determined experimentally $(\tilde{\theta})$ is the temperature at which $A_{2}=0$, where $A_{2}$ is the second virial coefficient of polymer molecules. At this temperature, it has been known that $\alpha=1^{1}$. From eq $1,(\theta-\tilde{\theta})=\left(v_{3} / v_{0} N^{1 / 2}\right) \tilde{\theta}$, then eq 1 is rewritten as

$$
\alpha^{5}-\alpha^{3}=\tilde{v}_{2} N^{1 / 2}+v_{3}\left(\alpha^{-3}-1\right),
$$

where

$$
\tilde{v}_{2}=\left(v_{0}+v_{3} N^{-1 / 2}\right)(1-\tilde{\theta} / T) .
$$

Curves for $\alpha^{2}$ vs. $\tilde{v}_{2} N^{1 / 2}$ calculated by eq 7 are shown in Figure 2.

The change in $\left\langle S^{2}\right\rangle$ accompanying the decrease of temperature from $T>\tilde{\theta}$ to $T<\tilde{\theta}$ has been known as the coil-globule transition. The

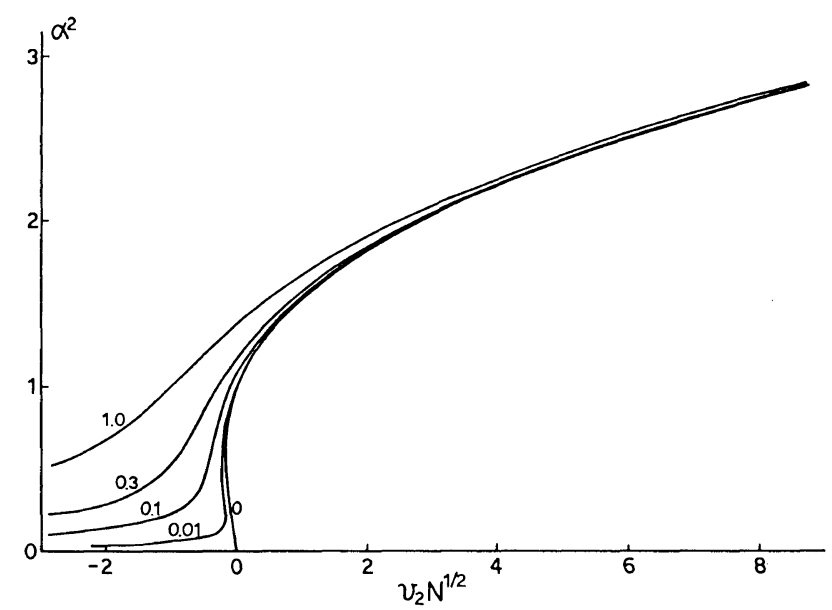

Figure 1. Calculated values of $\alpha^{2}$ as function of $v_{2} N^{1 / 2}$ using eq 1: the numbers on the curves are the values of $v_{3}$. 


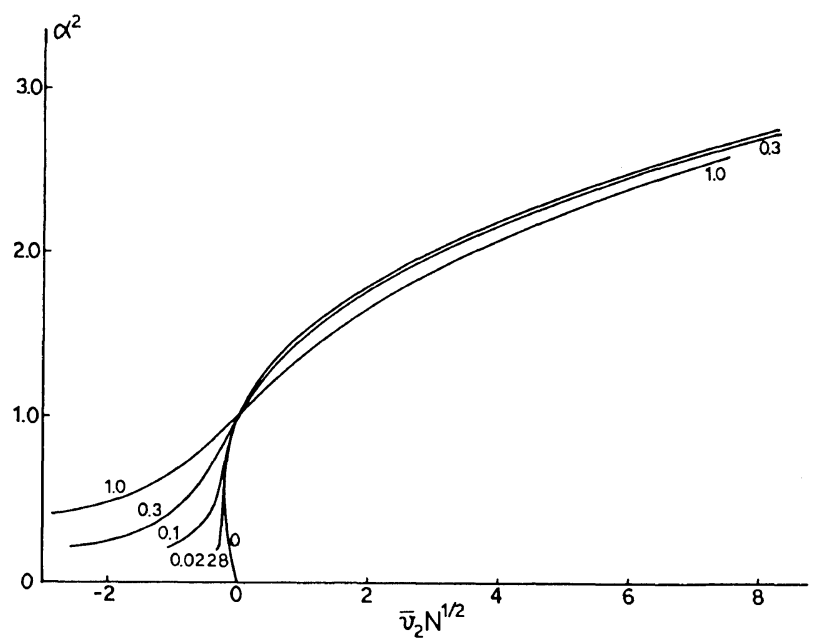

Figure 2. Calculated values of $\alpha^{2}$ as function of $\tilde{v}_{2} N^{1 / 2}$ using eq 7: the numbers on the curves are the values of $\tilde{v}_{3}$.

discontinuous transition by eq 7 takes place only in the range of $v_{3}<0.0228^{3}$ as shown in the Figure 2. However, the virial coefficients higher than the third were neglected in the derivation of eq 1 . This neglect is correct when $\varepsilon>0$ in the relation $\left\langle S^{2}\right\rangle \sim N^{1+\varepsilon}$, but when $\left\langle S^{2}\right\rangle \sim N^{2 / 3}$, all the higher virial coefficients become effective. ${ }^{4}$ As a result, the physical meaning of $v_{3}$ is vague in the range of $T<\theta$. However, the sharpness in the transition depends on the value of $v_{3}$.

\section{A SEMI-THEORETICAL EQUATION}

Although eq 7 is equivalent to eq 1 , the former is more complex than the latter in checking up with experimental results. Theoretically, $\tilde{\theta}$ depends on $N^{-1 / 2}$, since the segmental parameter $\theta$ is assumed to be constant, and it is believed $v_{0}$ and $v_{3}$ are the same order. But this dependence has not been reported experimentally, and $\tilde{\theta}$ is treated as a constant, usually. This discrepancy may be caused from the incorrect estimation of the three body interaction in the theory. Then we assume that $\tilde{\theta}$ and $v_{0}+v_{3} N^{-1 / 2}$ are constant in the experimental ranges of the molecular weight and $T$, and propose the next semi- theoretical equation,

$$
\alpha^{5}-\alpha^{3}=\tilde{v}_{0 M}(1-\tilde{\theta} / T) M^{1 / 2}+\tilde{v}_{3}\left(\alpha^{-3}-1\right)
$$

where $M$ is the molecular weight (Hereafter, the scale changes from $N$ to $M$, since $N$ can not be determined experimentally), and $\tilde{v}_{0 M}$ and $\tilde{v}_{3}$ are the phenomenological constant determined experimentally. $\tilde{v}_{0 \mathrm{M}}$ and $\tilde{v}_{3}$ depend not only on $v_{0}$ and $v_{3}$, but also on the range of $M$ used in experiments (especially the lower limit of $M$, since eq 7 is valid in the case $N \gg 1$ ). Although the applicable range of this approximation depends on the nature of the systems, eq 9 can be used for the analysis of the $\alpha-M$ and/or $\alpha-T$ relations having only two parameters and the standard point at $(1-\tilde{\theta} / T) M^{1 / 2}=0$, $\alpha=1$. In the following, we verify eq 9 experimentally.

\section{EXPERIMENTAL}

Sample polymers were monodisperse standard polystyrenes prepared by Toyo Soda Manufacturing Co., Ltd.: the sample symbols (the weight average molecular weights) were F4 $\left(4.28 \times 10^{4}\right), \quad$ F20 $\left(18.6 \times 10^{4}\right), \quad$ F40 $\left(42.2 \times 10^{4}\right), \quad F 80 \quad\left(77.5 \times 10^{4}\right)$, and F126 $\left(126 \times 10^{4}\right)$. The samples were used after 
freeze-drying without further purification. Cyclohexane, methylcyclohexane and benzene were used as solvents. They (special grade) were purified by the usual methods. Solutions were prepared at about $50^{\circ} \mathrm{C}$ for the cyclohexane and benzene solutions and at about $90^{\circ} \mathrm{C}$ for the methylcyclohexane solution. The solvents and solutions were optically clarified with a $0.20 \mu \mathrm{m}$ membrane filter (Toyo Roshi Co., Ltd., TM-4P). Light scattering measurements were carried out with the home made differential light scattering photometer described in detail in a separate paper. ${ }^{7}$ Measurements of the cyclohexane and methylcyclohexane solutions were made by cooling at a rate of $0.1 \mathrm{~K} \mathrm{~min}^{-1}$ from 55 and $90^{\circ} \mathrm{C}$, respectively. Measurements of the benzene solution were made at a constant temperature, $30^{\circ} \mathrm{C}$.

\section{RESULTS AND ANALYSIS}

Experimental results are summarized in Figures 3-8. The plots of $\alpha^{2} v s .(1-\tilde{\theta} / T) M^{1 / 2}$ are shown in Figures 3 and $4.34 .5^{\circ} \mathrm{C}$ and $66.8^{\circ} \mathrm{C}$ were used as the value of $\tilde{\theta}$ for the cyclohexane and methylcyclohexane solutions, respectively. In Figure 3, data by Miyaki and Fujita $^{8}$ are shown together. The curves in Figures 3 and 4 represent eq 9. The values of the phenomenological constants determined so as to best fit the experimental results are $\tilde{v}_{0 M}=$ 0.0102 and $\tilde{v}_{3}=0.18$. These values are common to both the cyclohexane and methylcyclohexane solutions and also represents the Miyaki-Fujita's data fairly well.

In Figure 5, the plot for $\alpha^{2} v s . M^{1 / 2}$ of the benzene solution (including data by Miyaki, Einaga, and Fujita ${ }^{9}$ ) are shown, where the

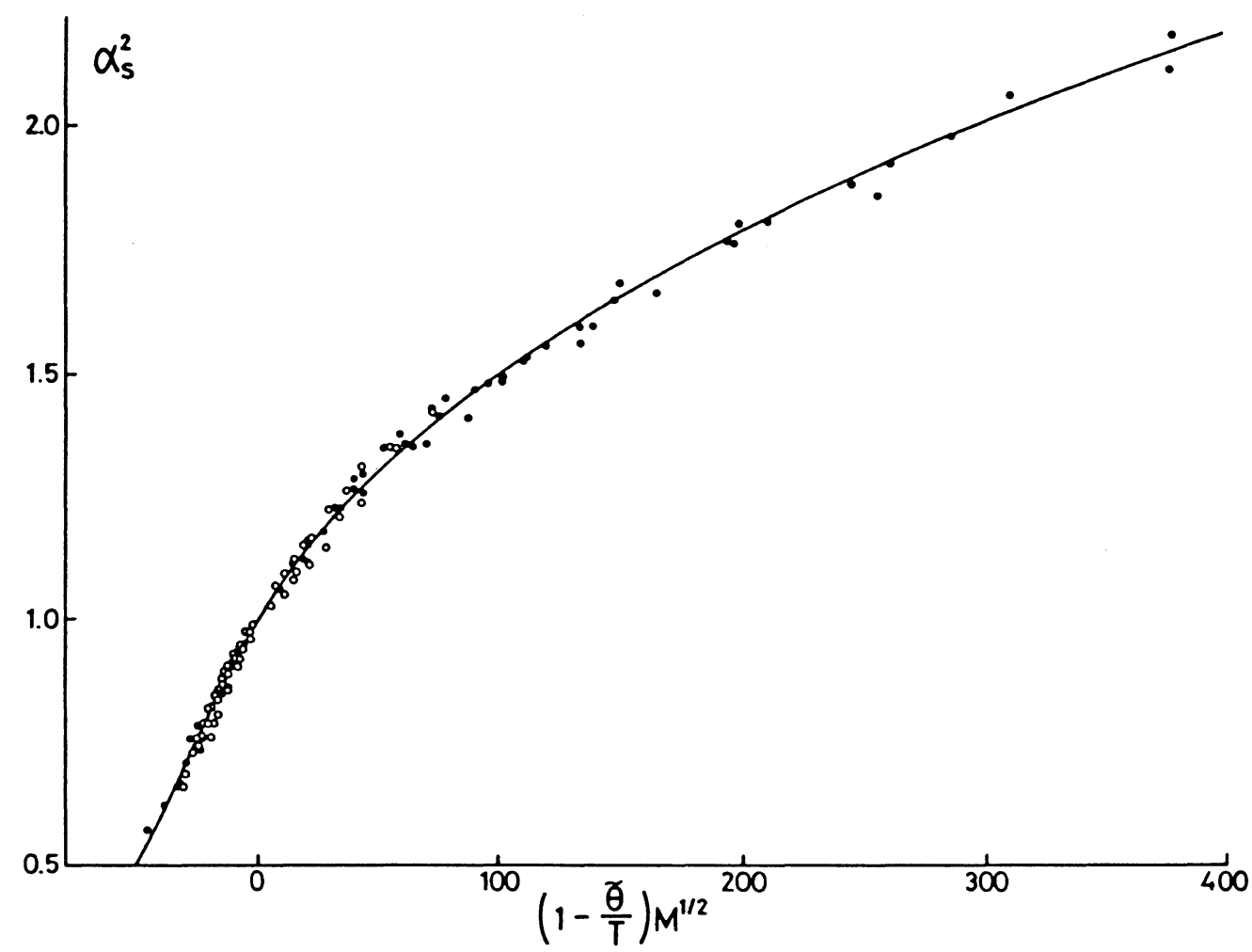

Figure 3. Plots of $\alpha_{\mathrm{S}}^{2} v s$. $(1-\tilde{\theta} / T) M^{1 / 2}$ for polystyrene in cyclohexane: (O), Present work; (O), Miyaki and Fujita. ${ }^{8}$ The solid curve is calculated using eq 9 with $\tilde{v}_{0 M}=0.0102$ and $\tilde{v}_{3}=0.18$. 
value of $\left\langle S^{2}\right\rangle$ in the cyclohexane solution at $34.5^{\circ} \mathrm{C}$ is used as the value of $\left\langle S^{2}\right\rangle_{0}$. The curve in the figure represents eq 9 , in which the value of $\tilde{v}_{0 \mathrm{M}}$ and $\tilde{v}_{3}$ are common to Figures 3 and 4 . From this curve, $140 \mathrm{~K}$ is given as $\tilde{\theta}$ of the benzene solution. Flory ${ }^{10}$ has obtained $100 \mathrm{~K}$ for this temperature from viscosity data.

In Figure 6, the plot for $\alpha^{2} v s .(1-\tilde{\theta} / T) M^{1 / 2}$ of the cyclohexane and benzene solutions are summarized. The curve is again eq 9 with $\tilde{v}_{0 \mathrm{M}}=0.0102$ and $\tilde{v}_{3}=0.18$, showing that eq 9 represents the experimental results well in the whole range of $(1-\tilde{\theta} / T) M^{1 / 2}$. The differences between $\tilde{\theta}$ and $\theta$ estimated under the assumptions of $\tilde{v}_{0 \mathrm{M}} M^{1 / 2}=v_{0} N^{1 / 2}$, and $\tilde{v}_{3}=v_{3}$ are about $5^{\circ} \mathrm{C}$ at $M=10^{6}$, and $15^{\circ} \mathrm{C}$ at $M=10^{5}$. This molecular weight dependence is large in comparison with known results. This large

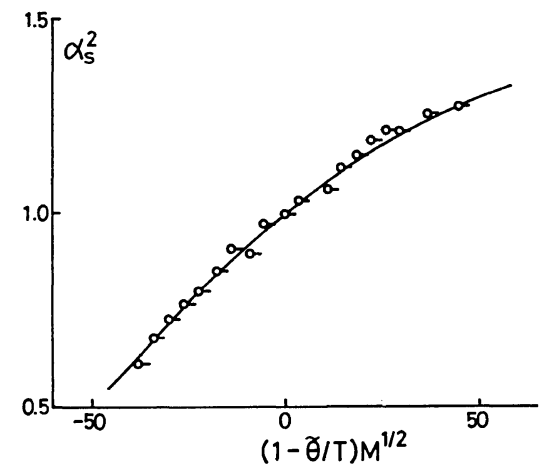

Figure 4. Plots of $\alpha_{\mathrm{S}}^{2} v s$. $(1-\tilde{\theta} / T) M^{1 / 2}$ for polystyrene in methylcyclohexane. The solid curve is calculated using eq 9: $\tilde{v}_{0 \mathrm{M}}$ and $\tilde{v}_{3}$ are the same as in Figure 3. difference is mainly due to the overestimation of $v_{3}$. In eq 7 , the term related to $v_{3}$ is negligible in the range of $T>\tilde{\theta}$, but becomes dominant in the range of $T<\tilde{\theta}$. Then $\tilde{v}_{3}$ is exclusively determined by the data in the range of $T<\tilde{\theta}$. As discussed in the theoretical background section, the terms related to higher than $v_{3}$ are relevant in the range of $T<\tilde{\theta}$, especially in the case of finite $M$ range. So $\tilde{v}_{3}$ is obtained as a "renormalized" constant including the related terms higher than $v_{3}$, and $v_{3}$ is smaller than $\tilde{v}_{3}$. On the other hand, $\tilde{\theta}$ depends on "bare" $v_{3}$ since higher terms can be negligible even at near $\theta$. However, in the range of $T<\tilde{\theta}$, the nature of system depends on the "renor-

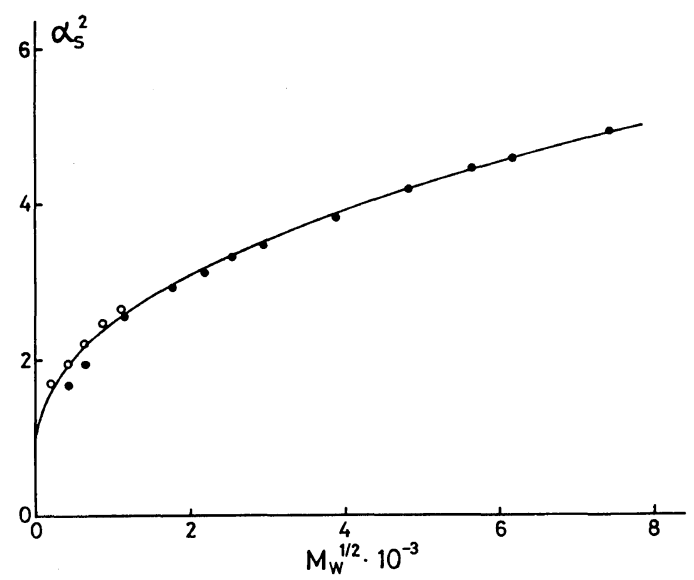

Figure 5. Plots of $\alpha_{S}^{2} v s . M^{1 / 2}$ for polystyrene in benzene: $(\bigcirc)$, Present work; (O), Miyaki et al. ${ }^{9}$ The solid curve is calculated using eq 9 with $\tilde{\theta}=140 \mathrm{~K}: \tilde{v_{0 M}}$ and $\tilde{v_{3}}$ are the same as in Figure 3.

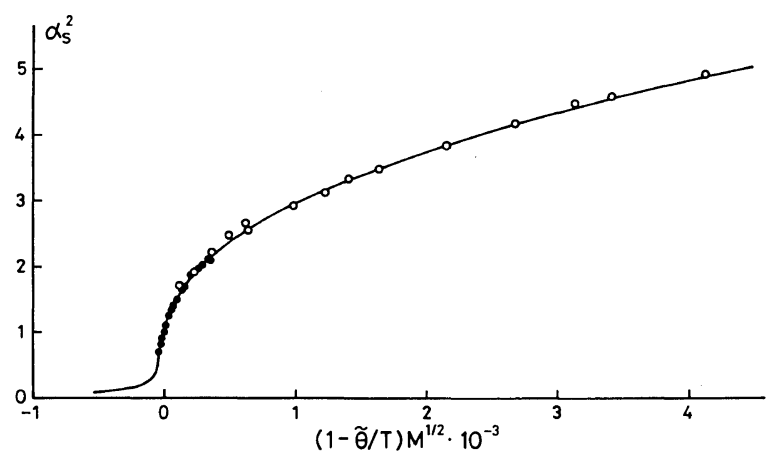

Figure 6. Plots of $\alpha_{\mathrm{S}}^{2} v s .(1-\tilde{\theta} / T) M^{1 / 2}$ for polystyrene in benzene and cyclohexane: $(\bigcirc)$, benzene; $(\bigcirc)$, cyclohexane. The solid curve is calculated using eq 9: $\tilde{v}_{0 \mathrm{M}}$ and $\tilde{v}_{3}$ are the same as in Figure 3 . 


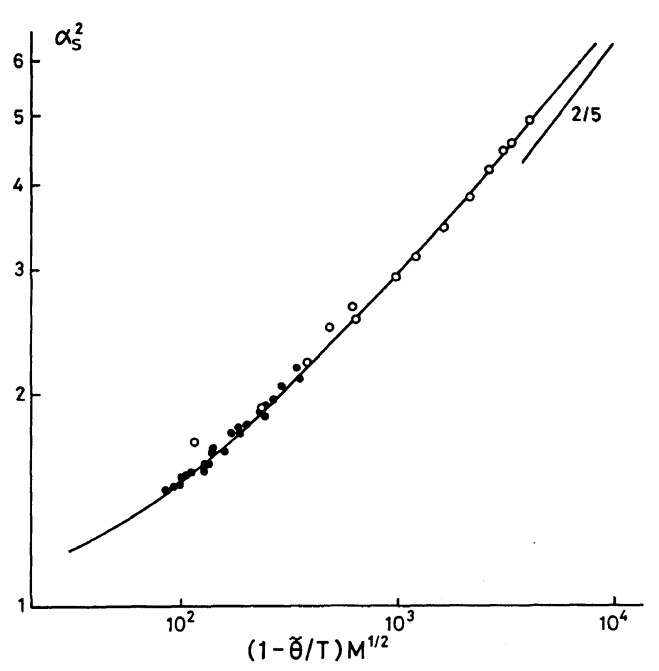

Figure 7. Double logarithmic plots of $\alpha_{\mathrm{S}}^{2} v s$. $(1-\tilde{\theta} / T) M^{1 / 2}$ for polystyrene in benzene and cyclohexane: the symbols and the curve are the same as in Figure 6.

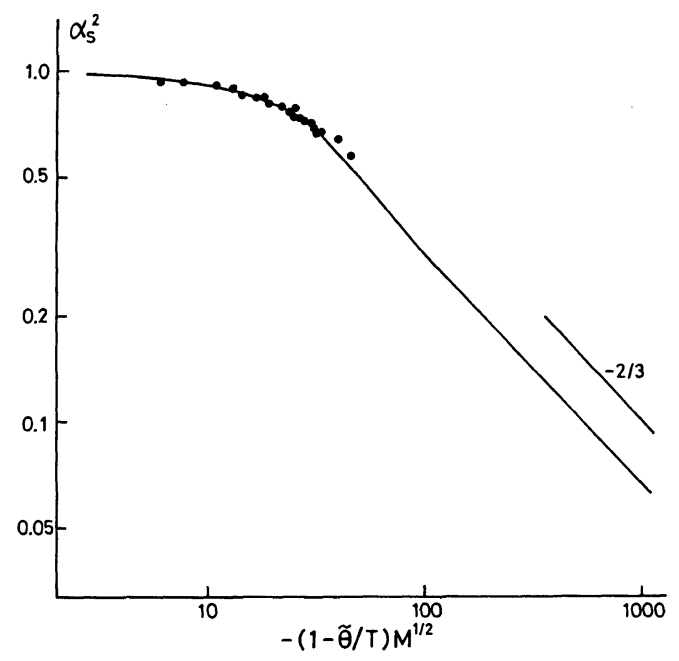

Figure 8. Double logarithmic plots of $\alpha_{S}^{2} v s$. $-(1-\tilde{\theta} / T) M^{1 / 2}$ for polystyrene in benzene and cyclohexane: the symbols and the curve are the same as in Figure 6.

malized" $\tilde{v}_{3}$, especially in the range of finite $M$.

To confirm the limiting exponent, the double logarithmic plots for $\alpha^{2}$ vs. (1$\tilde{\theta} / T) M^{1 / 2}(\equiv Z)$ are shown in Figures 7 and 8. In $Z>0$, the relation $\alpha^{2} \sim Z^{2 / 5}$ or $\left\langle S^{2}\right\rangle \sim(1-\tilde{\theta} / T)^{2 / 5} M^{6 / 5}$ was confirmed, but in $Z<0$, the limiting relation $\alpha^{2} \sim(-Z)^{-2 / 3}$ could not be confirmed since the experimental range of $Z$ is not sufficient. As shown in Figure 8, eq 9 predicts that the order of $10^{2}$ is necessary for the value of $Z$ to confirm the limiting exponent. For the cyclohexane solution of $M=$ $25 \times 10^{6}$, the value of $T$ for $Z=-1 \times 10^{2}$ is $28.5^{\circ} \mathrm{C}$. If the phase separation can be avoided, higher $M$ samples are more desirable.

\section{On the Coil-Globule Transition}

On the sharpness of the coil-globule transition, two opposite conclusions have been reported experimentally.

Sun, Nishio, Swislow, and Tanaka ${ }^{11}$ measured a sample $M=26 \times 10^{6}$ by analysis of the angular dependence of the autocorrelation function of light scattered from the solution. They used very dilute solutions, $3 \sim 0.03$ $\mu \mathrm{g} \mathrm{ml}^{-1}$, and measured down to $25^{\circ} \mathrm{C}$. They concluded that the sharp coil-globule transition was observed and this transition was in good agreement with the prediction by the mean field theory.

On the other hand, Nierlich, Cotton, and Farnoux ${ }^{12}$ measured a low molecular weight sample $M=2.9 \times 10^{4}$ by neutron scattering. They observed a cross-over from the coil state to the globule state and obtained the limiting exponent, $-2 / 3$, in the temperature range of $11.2^{\circ} \mathrm{C}-18^{\circ} \mathrm{C}$. However, this temperature range corresponds to the order of $Z \sim-10^{1}$ for their sample. Miyaki and Fujita ${ }^{8}$ measured samples up to $M=32 \times 10^{6}$ systematically. They could not observe the sharp transition. Slagowski, Tsai, and McIntyre ${ }^{13}$ measured a sample $M=44 \times 10^{6}$. However, no conclusion was given since the lowest measured temperature was $34^{\circ} \mathrm{C}$.

In Figure 9, experimental results for $\ln \left\langle S^{2}\right\rangle$ vs. $\ln M$ by us, Miyaki-Fujita, Slagowski et al., Sun et al., and Nierlich et al. are summarized. In the figure, the values of $\left\langle S^{2}\right\rangle$ for cited temperatures have been obtained by interporation from the original data and were shifted 


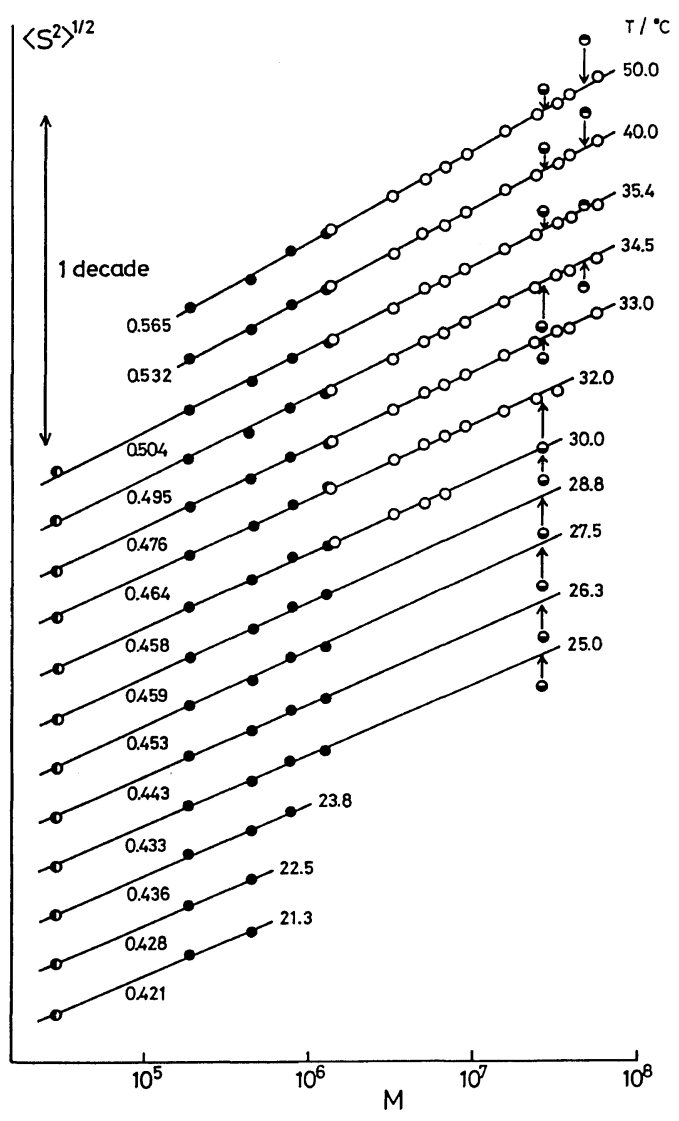

Figure 9. Double logarithmic plots of $\left\langle S^{2}\right\rangle^{1 / 2} v s . M$ for polystyrene in cyclohexane: $(\bigcirc)$, Present work; $(O)$, Miyaki and Fujita ${ }^{8} ;(\ominus)$, Sun et al. ${ }^{11} ;(\bigcirc)$, Nierlich et al. ${ }^{12} ;(\Theta)$, Slagowski et al. ${ }^{13}$ : the values of $\partial \ln \left\langle S^{2}\right\rangle^{1 / 2} /$ $\partial \ln M$ are indicated on the right hand side of the lines: for clarity of plotting, ordinates of lines have been shifted suitably.

suitably for clarity of plotting. Numerals on the left side of the figure are the values of $\partial \ln \left\langle S^{2}\right\rangle / \partial \ln M$.

The values of $\left\langle S^{2}\right\rangle$ at the same temperature by us, Miyaki-Fujita and Nierlich et al. are on the same lines. On the other hand, the values by Slagowski et al. and Sun et al. are larger than the indicated lines in the range above $35^{\circ} \mathrm{C}$ and smaller in the range below $35^{\circ} \mathrm{C}$. On these lines, the values of $\partial \ln \left\langle S^{2}\right\rangle / \partial \ln M$ gradually change from 0.565 to 0.421 with the decrease of temperature from $50^{\circ} \mathrm{C}$ to $21.3^{\circ} \mathrm{C}$.
In the present analysis, the value of $\tilde{v}_{3}$ was 0.18 . For this value of $\tilde{v}_{3}$, the extrapolation of eq 9 does not give the sharp transition and $\left\langle S^{2}\right\rangle$ decreases gradually with the decrease of temperature. From this point and the consideration on Figure 9, we conclude that the coil-globule transition is not a phase transition type but a cross-over type at least in the polystyrene-cyclohexane solution, in contrast to the result by Sun et al.

In the above discussions, studies on the hydrodynamic radius were omitted, since their experimental and theoretical bases differ from the Rayleigh scattering. However, from the quasi-elastic experiments, Perzynski, Adam, and Delsanti ${ }^{14}$ have concluded that there is no evidence of an asymptotic collapsed regime for $((\tilde{\theta}-T) / T) M^{1 / 2} \leq 35$. Recently, from the ultracentrifugal analysis, Vidaković and Rondelez $^{15}$ have shown that the cross over between the coil and globule regimes is smooth and continuous. These results agree with our conclusion.

Acknowledgement. This work was partially supported by a Grant-in-Aid for Scientific Research from the Ministry of Education, Science and Culture of Japan, for which the authors are very grateful.

\section{REFERENCES}

1. P. G. deGennes, "Scaling Concepts in Polymer Physics," Cornell University Press, Ithaca, 1979, Chapter 1 .

2. T. Oyama and Y. Oono, J. Phys. Soc. Jpn., 44, 1348 (1977).

3. O. B. Ptitsyn, A. K. Kron, and Yu. Ye. Eizner, J. Polym. Sci., C, 16, 3509 (1968).

4. T. Oyama and K. Shiokawa, Polym. J., 12, 507 (1980).

5. I. C. Sanchez, Macromolecules, 12, 980 (1979).

6. M. Daoud and G. Jannink, J. Physique, 37, 973 (1976).

7. T. Oyama, K. Shiokawa, and K. Baba, Polym. J., 13, 821 (1981).

8. Y. Miyaki and H. Fujita, Polym. J., 13, 749 (1981).

9. Y. Miyaki, Y. Einaga, and H. Fujita, Macromolecules, 11, 1180 (1978).

10. P. J. Flory, "Principles of Polymer Chemistry," 


\section{T. Oyama, K. Shiokawa, and K. Baba}

Cornell University Press, Ithaca, 1953, Chapter 14.

11. S. Sun, I. Nishio, G. Swislow, and T. Tanaka, $J$. Chem. Phys., 73, 5971 (1980).

12. M. Nierlich, J. P. Cotton, and B. Farnoux, J. Chem. Phys., 69, 1379 (1978).

13. E. Slagowski, B. Tsai, and D. McIntyre,
Macromolecules, 9, 687 (1976).

14. P. Perzynski, M. Adam, and M. Delsanti, J. Physique, 43, 129 (1982).

15. P. Vidaković and F. Rondelez, Macromolecules, 17, 418 (1984). 